\title{
Schedules in a Temporal Interpretation of Modals
}

\section{TIM FERNANDO}

Trinity College Dublin

\begin{abstract}
Eventualities and worlds are analysed uniformly as schedules of certain descriptions of eventuality-types (reversing the reduction of eventuality-types to eventualities). The temporal interpretation of modals in Condoravdi 2002 is reformulated to bring out what it is about eventualities and worlds that is essential to the account. What is essential, it is claimed, can be recovered from schedules that may or may not include worlds.
\end{abstract}

\section{INTRODUCTION}

Just as semantic accounts of modality commonly invoke possible worlds, theories of temporality (concerning, for instance, aspect) often appeal to eventualities. But what are eventualities? And what are worlds? The present work analyses eventualities and worlds uniformly as certain relations $s \subseteq$ TI $\times$ ED between a set Tr of times $t$ and a set ED of eventuality-descriptions $\varphi$, with

$$
s(t, \varphi) \text { pronounced ' } s \text { schedules } \varphi \text { at } t .
$$

Insofar as eventuality-descriptions apply to eventuality-types, we may call $s$ a schedule of eventuality-types. Exactly what eventualitydescriptions are and how they pick out eventuality-types depend on the application at hand: the fragment of English to be analysed, and the bit of reality that is conceptualised (to serve that end). In particular, we may derive ED from certain words and phrases under consideration, while basing eventuality-types on additional conceptualisations of, for instance, time.

A concrete and illuminating illustration is provided by the temporal interpretation of modals in Condoravdi 2002, henceforth CON2. Some sentences with which CON2 is concerned are listed in (1).

(1) a. He might be here right now.

b. He might be here any day now.

c. He might be here next week.

d. ${ }^{*} \mathrm{He}$ might be here yesterday. 
The oddness of (1d), marked by ${ }^{*}$, is broadly compatible with the idea of historical necessity (e.g. Thomason 1984), according to which the past is settled, and only the present and future are open to branching (whence the acceptability of $(1 \mathrm{a}-\mathrm{c})$ ). But if there are no might's about the past, then how do we explain (2)?

(2) She might have won.

What (2) has, which each sentence in (1) lacks, is the perfect (have -en), an analysis of which leads CoN2 to two readings of (2), given in (3).

(3) a. For all we know now, she might have won.

b. She might have at an earlier point won.

Con2 defines operators for the perfect and for might, deriving (3a) from the scoping (4a), and (3b) from (4b).

(4) a. MIGHT (PERF (she-win))

b. PERF (MIGHT (she-win))

Flying against the surface form (4a) of (2), the scoping of the perfect over might in (4b) is not uncontroversial. It is, however, crucial in CoN2 for imposing historical necessity on $(3 b) /(4 b)$ relative to a notion of history shifted towards an earlier point in the past. But is (4b) based on a flawed interpretation of the perfect? To understand this question, let us examine some of the assumptions underlying Con2.

CON2 draws on a generous inventory of worlds, states, events and times to form, on the one hand, eventive and stative properties, and, on the other hand, temporal properties. As made precise in section 3 below, eventive and stative properties serve as interpretations of eventuality-descriptions. To interpret the modals and the perfect, Con2 steps up to temporal properties, replacing the specific states and events in stative and eventive properties by times, alongside worlds that figure in all properties. Now, it is easy enough to convert a stative or eventive property to a temporal property by mapping states or events to their temporal trace. Going back from a temporal property to a stative or eventive one, however, runs into the problem that too many states and events may have the same temporal trace. For instance, does an interpretation of (5) as a temporal property allow us to extract the consequent state of Pat being away (left out from Con2, which focuses on the so-called existential perfect)?

(5) Pat has left.

And if we were to sharpen the temporal property interpreting PERF(A) to a stative property, could PERF still scope over MIGHT 
Table 1. From temporal properties to schedules in 3 steps.

\begin{tabular}{lll}
\hline Section 2 & Given: temporal property (from Con2) & $\varphi(w)(t)$ \\
Section 3 & Step 1: turn world $w$ into schedule $s_{w}$ (satisfies $\models$ ) & $s_{w}, t \models \varphi$ \\
Section 4 & Step 2: generalize $s_{w}$ to smaller schedules $s$ (forces $\Vdash$ ) & $s \Vdash t, \varphi$ \\
Section 5 & Step 3: reconstruct $s_{w}$ from (generic) set G of schedules & $s_{w}=\bigcup^{G}$ \\
\hline
\end{tabular}

as in (4b)? Not if PERF were to require states or events (in its inputs), whereas MIGHT returns times (in its outputs). Under the reformulation below, the opposition between temporal properties and stative/ eventive properties evaporates. The world-time pairs in temporal properties become schedules, encompassing worlds and eventualities alike. (4b) is kept viable, and so the reader wishing to rule out (4b) must seek other grounds for doing so.

The reformulation of CON2 below is intended as a first step at pinning down the semantic entities CoN2's modals and perfect characterise - a first step, that is, to isolating what Schubert (2000) calls characterised situations. The main thrust is to strip world-time pairs down to the essentials - or, at least, to schedules, from which, it is claimed, the essentials can be extracted. This is carried out below in three steps, outlined in Table 1.

We proceed in the next section, section 2, from the semantic set-up in CoN2, converting worlds into schedules in section 3. We introduce schedules other than those induced by worlds in section 4 , before making do without world-induced schedules in section 5. Precisely what the symbols in Table 1 mean will be explained in due course. That said, let us note at the outset that appeals to forcing $\Vdash$ (as in sections 4 and 5) are not new in philosophical semantics, stretching at least as far back as van Fraassen (1969). Forcing lurks at the background of the data semantics of Veltman (1984), where its impact is diminished by the failure of (what is termed there) 'stability.' Stability relates to (3) above roughly as follows. (3b) is stable insofar as it is tenable even if we accept that she did not win.

(6) She might have at an earlier point won (had she followed my advice ...), but she didn't.

By contrast, accepting she did not win makes (3a) untenable and, in that sense, unstable.

(7) a. She didn't win. *But for all we know now, she might have.

b. She didn't win. But she might have (had she ...). 
More formally, stability coincides in section 4 below with the persistence of $\Vdash \vdash$ relative to the subset relation $\subseteq$ on schedules $s, s^{\prime}$

$$
s \Vdash t, \varphi \quad \text { and } \quad s \subseteq s^{\prime} \quad \text { implies } \quad s^{\prime} \Vdash t, \varphi \text {. }
$$

Persistence is indispensable to the application we shall make of forcing (e.g. Proposition 3, section 5). Now, while (3b)/(6), (7b) may pose no problem for persistence, (3a)/(7a) is a different matter. Suppose $s$ forced (3a) and $s^{\prime}$ encoded she lost, whereas $s$ did not. Then surely $s^{\prime}$ could not force (3a)?

In fact, it could, provided we analyze epistemic might not as in Veltman 1984's data semantics, but more along the lines of Veltman's (1996) update semantics. Dropping $t$ for the sake of clarity (at the cost of correctness) and writing $\langle e\rangle \varphi$ for 'might epistemically $\varphi$,' let

$$
s \Vdash\langle e\rangle \varphi \quad \text { iff } \quad\left(\exists s^{\prime} \in \Sigma\right) s^{\prime} \Vdash \varphi
$$

for some set $\Sigma$ of schedules specifying the epistemic possibilities of $\Vdash$ (without regard to $s$ ). As $s$ appears only in the left side (not the right) of the biconditional, we can restore $t$ to get (8), ${ }^{1}$ making persistence with respect to $\langle\mathrm{e}\rangle \varphi$ unproblematic.

(8) For all schedules $s, s^{\prime}$ in the domain of $\Vdash$,

$$
s \Vdash t,\langle\mathrm{e}\rangle \varphi \quad \text { iff } \quad s^{\prime} \Vdash t,\langle\mathrm{e}\rangle \varphi \text {. }
$$

What then becomes of the instability in (7a)? Rather than analysing (7a) in terms of a single non-persistent forcing relation $\Vdash$, we appeal to context change of the kind advocated in Veltman (1996). The first sentence of (7a), she didn't win, changes the epistemic base $\Sigma$ to $\Sigma^{\prime}$, effectively inducing a new forcing relation $\Vdash^{\prime}$, relative to which (3a) fails (whether or not it holds for the initial relation $\Vdash$ ). ${ }^{2}$

Notice that if we are to make sense of discourses such as $(7 \mathrm{~b})$, the first sentence in which rules out possibilities entertained in the second, we must keep the epistemic base for $(3 a) /(4 a)$ separate from the modal base for $(3 \mathrm{~b}) /(4 \mathrm{~b})$, called metaphysical in Con2. ${ }^{3}$ Accordingly, we shall

\footnotetext{
${ }^{1}$ This would suggest that the situation characterised by $\langle\mathrm{e}\rangle \varphi$ is not so much a schedule $s$ that forces $\langle e\rangle \varphi$ but rather the set $\left\{s^{\prime} \in \Sigma \mid s^{\prime} \Vdash \varphi\right\}$ of schedules in $\Sigma$ that force $\varphi$.

${ }^{2}$ My apologies for the notational clash with Veltman 1996, where might $\phi$ is described as nonpersistent relative to a predicate $\Vdash$ that takes on its left side not $s$, but rather a state $\sigma$ corresponding (here) to a set such as $\Sigma$ of $s$ 's. My $\Vdash$ is just a slice of Veltman's $\Vdash$, fixed by a choice of $\sigma / \Sigma$ in the background. (In saying this, I am putting aside times $t$ that appear to the right of my $\Vdash$, but not Veltman's. Variations in $t$ should, of course, not be confused with Veltman's updates of $\sigma / \Sigma$.)

${ }^{3}$ We must, so to speak, immunise metaphysical might from updates that infect epistemic might.
} 
assume $\Vdash$ comes with two sets $\Sigma_{\mathrm{e}}$ and $\Sigma_{\mathrm{m}}$ of schedules specifying the epistemic and metaphysical possibilities, respectively. To avoid cluttering the notation, we will refrain from hanging the sets $\Sigma_{\mathrm{e}}, \Sigma_{\mathrm{m}}$ as subscripts on $\Vdash$. Such a practice would be useful were we to encode a dynamic interpretation of conjunction involving changes to $\Sigma_{\mathrm{e}}$ (and possibly also to $\Sigma_{\mathrm{m}}, s$ and $t$ ). But the present paper stops short of that, keeping $\Sigma_{\mathrm{e}}$ and $\Sigma_{\mathrm{m}}$ frozen. ${ }^{4}$ Holding $\Sigma_{\mathrm{e}}, \Sigma_{\mathrm{m}}$ constant, we will have enough to do sorting out complications involving time, the perfect and metaphysical might (omitted in Veltman 1984, 1996).

\section{TEMPORAL PROPERTIES IN CON2}

The semantic set-up in Con2 takes the following ingredients for granted:

(i) a set $\mathrm{PT}$ of temporal points/moments/instants linearly ordered by $<$, and a set TI $\subseteq$ Pow $\left(\mathrm{PT}_{\mathrm{T}}\right)-\{\emptyset\}$ of times consisting of non-empty subsets $t$ of $\mathrm{PT}$ such that for every $z \in \mathrm{P}_{\mathrm{T}}$,

$z \in t \quad$ whenever $x<z<y \quad$ for some $x, y \in t$

(that is, time is a non-empty $<$-interval)

(ii) sets $\mathrm{WO}, \mathrm{Ev}$ and $\mathrm{ST}$ of worlds, of events and of states, respectively, along with a function $\tau:(\mathrm{EV} \cup \mathrm{ST}) \times \mathrm{WO} \rightarrow(\mathrm{TI} \cup\{\emptyset\})$ that specifies the temporal trace $\tau(e, w) \in$ TI $\cup\{\emptyset\}$ of an event or state $e$ in world $w$, where

$\tau(e, w)=\emptyset \quad$ iff $\quad e$ is not realized in $w$

the intuition behind $\tau(e, w) \in$ Tr being that $e$ is a single token/ occurrence in $w$ (as opposed to a type that recurs in $w$ ).

Con2 calls a function $P$ from worlds

(i) eventive if for every world $w, P(w)$ is a unary predicate on events (so $P(w)(e)$ is either true or false for every event $e$ )

(ii) stative if for every world $w, P(w)$ is a unary predicate on states (so $P(w)(e)$ is either true or false for every state $e$ )

(iii) temporal if for every world $w, P(w)$ is a unary predicate on times (so $P(w)(t)$ is either true or false for every time $t)$

(iv) a property if $P$ is eventive or stative or temporal.

\footnotetext{
${ }^{4}$ Thus, other instances of instability noted in Veltman (1984) need not concern $\Vdash$, which in its static form, is not designed to cover all formulas that arise in natural language interpretation.
} 
To turn any property to a temporal property, a world-time pair $w, t$ is assigned sets $\operatorname{Ev}(w, t)$ and $\operatorname{ST}(w, t)$ of events and states as follows. An event is located at $w, t$ if its temporal trace in $w$ is contained in $t$

$$
\operatorname{Ev}(w, t)=\{e \in \operatorname{Ev} \mid \emptyset \neq \tau(e, w) \subseteq t\}
$$

whereas a state is located in $w, t$ if its temporal trace in $w$ overlaps with $t$

$$
\mathrm{ST}(w, t)=\{e \in \mathrm{ST} \mid \tau(e, w) \cap t \neq \emptyset\} .
$$

(Viewed from outside $t$, events give the impression of being bounded while states do not. Events occur, states hold.) A property $P$ is then mapped to the temporal property $\lambda w \lambda t \mathrm{AT}(t, w, P)$ by existentially quantifying over the events and states located in $w, t$

$$
\operatorname{AT}(t, w, P)= \begin{cases}(\exists e \in \operatorname{Ev}(w, t)) P(w)(e) & \text { if } P \text { is eventive } \\ (\exists e \in \operatorname{ST}(w, t)) P(w)(e) & \text { if } P \text { is stative } \\ P(w)(t) & \text { if } P \text { is temporal. }\end{cases}
$$

AT is used to formalize both the perfect and the modals. A function PERF mapping properties $P$ to temporal properties is defined by

$$
(\operatorname{PERF} P)(w)(t)=\left(\exists t^{\prime}<t\right) \operatorname{AT}\left(t^{\prime}, w, P\right)
$$

where the linear order $<$ on $\mathrm{PT}$ is extended to a relation on TI by quantifying universally over the points

$$
t<t^{\prime} \quad \text { iff } \quad(\forall x \in t)\left(\forall x^{\prime} \in t^{\prime}\right) x<x^{\prime} .
$$

To analyse modals, a modal base function $\mathrm{MB}$ is assumed that maps a world-time pair $(w, t)$ to a set of worlds, relative to which a function MIGHT $_{\mathrm{MB}}$ maps a property $P$ to the temporal property satisfying

$$
\left(\operatorname{MIGHT}_{\mathrm{MB}} P\right)(w)(t)=\left(\exists w^{\prime} \in \operatorname{MB}(w, t)\right) \operatorname{AT}\left(t_{\infty}, w^{\prime}, P\right)
$$

where (expanding time forward, as in Abusch 1998) ${ }^{5} t_{\infty}$ is the indefinite extension of $t$ to the future

$$
\{x \in \operatorname{TI} \mid(\exists y \in t) y \leqslant x\}
$$

\footnotetext{
${ }^{5}$ Gennari (2003) makes a claim related to the idea that modals expand time forward.
} 
(with $y \leqslant x$ abbreviating ' $y<x$ or $x=y$ '). To capture historical necessity, worlds are bundled at each time $t$ by an equivalence relation $\simeq_{t}$ (on Wo) satisfying (9).

(9) For every temporal property $\hat{P}$ of interest,

$$
\hat{P}(w)(t) \quad \text { iff } \quad\left(\forall w^{\prime} \simeq_{t} w\right) \hat{P}\left(w^{\prime}\right)(t) .
$$

The qualification 'of interest' in (9) is necessary to allow for branching in the future (i.e. beyond $t$ ); otherwise, $\simeq_{t}^{\prime}$ s satisfying (9) must be equality, in view of uninteresting temporal properties such as those given, for every world $w$, by

$$
\left(\forall w^{\prime} \in \mathrm{WO}\right)(\forall t \in \mathrm{TI}) \quad \hat{P}\left(w^{\prime}\right)(t) \text { iff } w^{\prime}=w .
$$

The metaphysical alternatives to $w$ at $t$ are restricted to worlds that share the same $t$-history as $w$.

(10) $\left(\forall w^{\prime} \in \operatorname{MB}(w, t)\right)\left(\forall t^{\prime}<t\right) w \simeq_{t^{\prime}} w^{\prime}$ for metaphysical MB.

Consequently, if the 'present perspective'

$\left(\operatorname{MIGHT}_{\mathrm{MB}}(\operatorname{PERF} P)\right)(w)(t)$

$$
=\left(\exists w^{\prime} \in \operatorname{MB}(w, t)\right)\left(\exists t^{\prime}<t\right) \operatorname{AT}\left(t^{\prime}, w^{\prime}, P\right)
$$

is to differ from (PERF $P))(w)(t)$, then $\mathrm{MB}$ had better not be metaphysical. Not so for the "past perspective"

$$
\begin{aligned}
& \left(\operatorname{PERF}\left(\operatorname{MighT}_{\mathrm{MB}} P\right)\right)(w)(t) \\
& \quad=\left(\exists t^{\prime}<t\right)\left(\exists w^{\prime} \in \operatorname{MB}\left(w, t^{\prime}\right)\right) \operatorname{AT}\left(t^{\prime}{ }_{\infty}, w^{\prime}, P\right)
\end{aligned}
$$

as PERF pushes $t$ back to $t^{\prime}$, which MIGHT then expands forward. Hence, CON2 disambiguates (2) by deriving the epistemic reading from (4a) and the metaphysical reading from (4b).

\section{A REFORMULATION IN TERMS OF SCHEDULES}

We are at Step 1 of Table 1, the point of which is to reformulate the temporal properties $\varphi(w)(t)$ from section 2 in terms of satisfaction

$$
s, t \models \varphi
$$

where $s$ is a schedule capturing $w$. To speak properly about schedules, we need to specify a set ED of eventuality-descriptions, relative to 
which schedules are subsets of $T_{I} \times$ ED. Given a set $E P$ of eventive and stative properties (as in section 2), let ED be the set of names $\dot{P}$ of $P \in E P$,

$$
\mathrm{ED}=\{\dot{P} \mid P \in E P\}
$$

with $\dot{P} \neq \dot{P}^{\prime}$ for $P \neq P^{\prime}$. Then every world $w$ induces the schedule

$$
s_{w, E P, \tau}=\{(t, \dot{P}) \mid P \in E P \text { and } \exists e[P(w)(e) \text { and } \tau(e, w)=t]\} .
$$

As is common in the literature (e.g. Dowty 1979), let us assume that states are divisible in the sense of (11).

(11) For all $e \in \mathrm{ST}, P \in E P, w \in \mathrm{WO}$ and $t \in \mathrm{T}$, if $P(w)(e)$ and $t \subseteq$ $\tau(e, w)$ then there is an $e^{\prime} \in \mathrm{ST}$ such that $P(w)\left(e^{\prime}\right)$ and $t=\tau\left(e^{\prime}, w\right)$.

Given (11), it is easy to prove

\section{Proposition 1.}

For all $P \in E P, w \in \mathrm{Wo}$ and $t \in \mathrm{T} \mathrm{I}$,

$$
\operatorname{AT}(t, w, P) \quad \text { iff } \quad\left(\exists t^{\prime} \subseteq t\right) s_{w, E P, \tau}\left(t^{\prime}, \dot{P}\right) .
$$

Let us treat ED as the set of atomic sentences in a language $\Phi \supseteq$ ED generated by the clause

$$
\text { whenever } \varphi \in \Phi, \text { so are : } \varphi_{\subseteq}, \operatorname{Perf}(\varphi),\langle\mathrm{m}\rangle \varphi,[\mathrm{m}] \varphi,\langle\mathrm{e}\rangle \varphi,[\mathrm{e}] \varphi .
$$

The intent is that $\varphi_{\subseteq}$ express the step from $\tau$ to AT in section 2 , and $\mathrm{m}$ and e label metaphysical and epistemic modals (with diamond/ $\exists$ and box $/ \forall^{6}$ forms $\langle\cdot\rangle$ and $[\cdot]$ ) respectively. To be more precise, let us agree that for $s \subseteq$ Ti $\times$ ED, $t \in$ Ti and $\varphi \in \Phi$,

(i) eventuality-descriptions are satisfied exactly if they are scheduled

$$
s, t \models \varphi \quad \text { iff } \quad s(t, \varphi) \quad \text { for } \varphi \in \mathrm{ED}
$$

(ii) $\varphi_{\subseteq}$ allows for temporal slack

$$
s, t \vDash \varphi \subseteq \quad \text { iff } \quad\left(\exists t^{\prime} \subseteq t\right) s, t^{\prime} \vDash \varphi
$$

\footnotetext{
${ }^{6}$ CON2 defines a universal variant of MIGHT, called WOLL, reformulated here in terms of square brackets $[\mathrm{m}],[\mathrm{e}]$.
} 
(iii) Perf pushes time back

$$
s, t \vDash \operatorname{Perf}(\varphi) \quad \text { iff } \quad\left(\exists t^{\prime}<t\right) s, t^{\prime} \vDash \varphi .
$$

Proposition 1 can then be restated as

$$
\mathrm{AT}(t, w, P) \quad \text { iff } \quad s_{w, E P, \tau}, t \models \dot{P}_{\subseteq}
$$

from which it follows that

$$
(\operatorname{PERF} P)(w)(t) \quad \text { iff } \quad s_{w, E P, \tau}, t \models \operatorname{Perf}\left(\dot{P}_{\subseteq}\right) .
$$

As for the modals, let us attend first to the time expansion $t_{\infty}$ from section 2 , defining $t^{\prime} \geqslant{ }_{\mathrm{o}} t$ (pronounced ' $t$ ' expands $t$ forward') as the conjunction

$$
\left(\forall x^{\prime} \in t^{\prime}\right)(\exists x \in t) x \leqslant x^{\prime} \text { and }(\forall x \in t)\left(\exists x^{\prime} \in t^{\prime}\right) x \leqslant x^{\prime} .
$$

Known as the Plotkin $\leqslant$-preorder (Plotkin 1983), $\geqslant_{\text {o }}$ compares both end points of open intervals $(l, r)$ and $\left(l^{\prime}, r^{\prime}\right)$

$$
\left(l^{\prime}, r^{\prime}\right) \geqslant_{\mathrm{o}}(l, r) \text { iff } \quad l \leqslant l^{\prime} \text { and } r \leqslant r^{\prime}
$$

so that for example, $(2,3) \geqslant_{\mathrm{o}}(0,1)$ and $(0,2) \geqslant_{\mathrm{o}}(0,1)$. It is easy to see that (12) holds.

$$
\begin{aligned}
\left(\operatorname{MIGHT}_{\mathrm{MB}} P\right)(w)(t) \quad \text { iff } \quad & \left(\exists w^{\prime} \in \operatorname{MB}(w, t)\right)\left(\exists t^{\prime} \geqslant_{\mathrm{o}} t\right) \\
& \operatorname{AT}\left(t^{\prime}, w^{\prime}, P\right)
\end{aligned}
$$

Next, let us spell out the modal base functions $\operatorname{MB}(w, t)$ above, given two sets $W_{\mathrm{m}}, W_{\mathrm{e}} \subseteq \operatorname{Pow}(\mathrm{Tr} \times \mathrm{ED})$ of schedules for the metaphysical and epistemic possibilities respectively. To impose historical necessity on the metaphysical alternatives, let $\approx_{t}$ hold between schedules that are the same up to times $<t$

$$
s^{\prime} \approx_{t} s \quad \text { iff } \quad\left(\forall t^{\prime}<t\right)(\forall \varphi \in \mathrm{ED}) s\left(t^{\prime}, \varphi\right) \text { iff } s^{\prime}\left(t^{\prime}, \varphi\right) .
$$

\footnotetext{
${ }^{7}$ Observe that if $t$ has left end point $l$, and $\{l\} \in$ TI. then we can make do in (12) with $t^{\prime} \geqslant\{l\}$ in place of $t^{\prime} \geqslant_{0} t$. But, as we cannot, in general, reduce $t$ to a point, and as it is not inconceivable that we may wish to eliminate the slack in AT (analysed in Proposition 1 via $\dot{P}_{\subseteq}$ ), I have kept the Plotkin pre-order above.
} 
The metaphysical alternatives are then defined from $\approx_{t}$ and $W_{\mathrm{m}}$ by strengthening (10) as follows

$$
m b_{\mathrm{m}}(s, t)=\left\{s^{\prime} \in W_{\mathrm{m}} \mid s^{\prime} \approx_{t} s\right\} .
$$

Reducing the epistemic alternatives to $W_{\mathrm{e}}$

$$
m b_{\mathrm{e}}(s, t)=W_{\mathrm{e}},
$$

we can, for $a \in\{\mathrm{e}, \mathrm{m}\}$, uniformly interpret $\langle a\rangle$ as quantifying existentially over modal alternatives

$$
s, t \vDash\langle a\rangle \varphi \quad \text { iff } \quad\left(\exists s^{\prime} \in m b_{a}(s, t)\right)\left(\exists t^{\prime} \geqslant_{\mathrm{o}} t\right) s^{\prime}, t^{\prime} \vDash \varphi
$$

and $[a]$ as quantifying universally

$$
s, t \models[a] \varphi \quad \text { iff } \quad\left(\forall s^{\prime} \in m b_{a}(s, t)\right)\left(\exists t^{\prime} \geqslant_{\mathrm{o}} t\right) s^{\prime}, t^{\prime} \models \varphi .
$$

Henceforth, we may assume that $\models \subseteq\left(\operatorname{Pow}\left(\mathrm{TI}_{\mathrm{I}} \times \mathrm{ED}\right) \times \mathrm{TI}\right) \times \Phi$ is determined by a choice $W_{\mathrm{m}}, W_{\mathrm{e}}$ of a pair of sets of schedules. Let us write $P$ for an element of ED (dropping the dot on $\dot{P} \in E P$ ) and write $w$ instead of $s$, construing worlds (from here on) as schedules. As hinted in the introduction above, ${ }^{8}$ a pair $(\varphi, t) \in \Phi \times$ Tr induces a change in the epistemic modal base from $W_{\mathrm{e}}$ to

$$
\left\{w \in W_{\mathrm{e}} \mid w, t \models \varphi\right\} .
$$

Whether the set $W_{\mathrm{m}}$ of metaphysical possibilities should be updated, I am less confident. It seems to me quite reasonable to equate $W_{\mathrm{m}}$ with the initial set of epistemic possibilities, and to assume that while $W_{\mathrm{e}}$ shrinks, $W_{\mathrm{m}}$ stays fixed-so that $W_{\mathrm{e}} \subseteq W_{\mathrm{m}}$. But I will not insist on that below.

\section{A PERSISTENT GENERALIZATION}

We are at Step 2 of Table 1, in which we consider schedules other than those induced by worlds, by adding schedules smaller than worldschedules. More precisely, given a set $W \subseteq P$ ow $($ TI $\times$ ED) of schedules

\footnotetext{
${ }^{8}$ We write $W_{\mathrm{m}}, W_{\mathrm{e}}$ instead of $\Sigma_{\mathrm{m}}, \Sigma_{\mathrm{e}}$ to distinguish satisfaction $\vDash$ (with which the $W$ 's are associated) from forcing $\Vdash$ (with which the $\Sigma$ 's are associated).
} 
(say, $W_{\mathrm{m}} \cup W_{\mathrm{e}}$ ), let $\downarrow W$ be the set $\cup_{w \in W} \operatorname{Pow}(w)$ of all subsets of schedules in $W$

$$
\downarrow W=\{s \subseteq \mathrm{TI} \times \mathrm{ED} \mid(\exists w \in W) s \subseteq w\} .
$$

What schedules are induced by worlds? Let us call a set $S$ of schedules an anti-chain if no two distinct elements in $S$ are related by $\subseteq$ that is, for all $s$, $s^{\prime} \in S$, if $s \subseteq s^{\prime}$ then $s=s^{\prime}$. We may assume that a set of worlds (with worlds construed as schedules) is an anti-chain, by arranging, for example, the eventuality descriptions ED to come with a 'negation' map ${ }^{-}: \mathrm{ED} \rightarrow \mathrm{ED}$ and excluding from schedules relations $s$ such that for some $t$ and $P$, both $s(t$, $P)$ and $s(t, \bar{P}) .{ }^{9}$ Now, the 'persistent generalisation' from which the present section gets its title is the definition of a forcing relation

$$
\Vdash \quad \subseteq \operatorname{Pow}\left(\mathrm{TI}_{\mathrm{I}} \times \mathrm{ED}\right) \times(\mathrm{TI} \times \Phi)
$$

from a pair $W_{\mathrm{m}}, W_{\mathrm{e}}$ of sets of schedules (determining a satisfaction relation $\vDash$ according to the previous section) such that

\section{Proposition 2.}

(a) For all $s, s^{\prime} \in \downarrow\left(W_{\mathrm{m}} \cup W_{\mathrm{e}}\right), t \in$ TI and $\varphi \in \Phi$,

$s \Vdash t, \varphi$ and $s \subseteq s^{\prime}$ implies $s^{\prime} \Vdash t, \varphi$.

(b) For all $w \in W_{\mathrm{m}} \cup W_{\mathrm{e}}, t \in$ TI and $\varphi \in \Phi$,

$$
w, t \vDash \varphi \quad \text { iff } \quad w \Vdash t, \varphi
$$

assuming $W_{\mathrm{m}} \cup W_{\mathrm{e}}$ is an anti-chain.

Part (a) of Proposition 2 is (as indicated in the introduction) what we mean by $\Vdash$ being persistent, while part (b) is the sense in which we get a generalization of $\models$. The shift in $t$ from the left of $\models$ to the right of $\Vdash$ is designed to isolate (and thereby highlight) the partial order $\subseteq$ on schedules (to the left of $\Vdash$ ). The idea is to reduce the schedules $s$ to the left of $\Vdash$ so that they have just enough weight to force the pairs $t, \varphi$ to the right of $\Vdash$. That is, having blown $W_{\mathrm{m}} \cup W_{\text {e }}$ apart into $\downarrow\left(W_{\mathrm{m}} \cup W_{\mathrm{e}}\right)$, we might seek a subset $B \subseteq \downarrow\left(W_{\mathrm{m}} \cup W_{\mathrm{e}}\right)$ of 'basic' schedules smaller than those in $W_{\mathrm{m}} \cup W_{\mathrm{e}}$, from which to reconstruct $\vDash$ according to (13).

\footnotetext{
${ }^{9}$ More specifically, we might pair each eventuality description $P$ with + (for truth) or - (for falsehood), defining $(P,+)=(P,-)$ and $\overline{(P,-)}=(P,+)$. The doubling of ED here corresponds in data semantics (Veltman 1984) to flipping $\Vdash$ for a notion of falsehood (complementing truth).

Notice that there is nothing anomalous about both $w\left(t, P_{\subseteq}\right)$ and $w\left(t, \bar{P}_{\subseteq}\right)$ holding. This explains the elimination in section 3 of the slack in AT, before its re-introduction via the mapping $\varphi \mapsto \varphi_{\subseteq}$.
} 
(13) For all $w \in W_{\mathrm{m}} \cup W_{\mathrm{e}}, t \in$ TI and $\varphi \in \Phi$,

$$
w, t \models \varphi \quad \text { iff } \quad(\exists s \in B)(s \subseteq w \text { and } s \Vdash t, \varphi) .
$$

More on (13) in the next section.

In the meantime, let us attend to the definition of $\Vdash$. The nonmodal clauses are as in (i)-(iii) of section 3

$$
\begin{array}{rll}
s \Vdash t, P & \text { iff } & s(t, P) \quad \text { for } P \in \mathrm{ED} \\
s \Vdash t, \varphi \subseteq & \text { iff } & \left(\exists t^{\prime} \subseteq t\right) s \Vdash t^{\prime}, \varphi \\
s \Vdash t, \operatorname{Perf}(\varphi) & \text { iff } & \left(\exists t^{\prime}<t\right) s \Vdash t^{\prime}, \varphi .
\end{array}
$$

For the modalities, we need to fix sets $\Sigma_{\mathrm{m}}$ and $\Sigma_{\mathrm{e}}$ of schedules, just as we did for $\models$ with $W_{\mathrm{m}}$ and $W_{\mathrm{e}}$. To establish Proposition 2, we must choose $\Sigma_{\mathrm{m}}=\downarrow W_{\mathrm{m}}$ and $\Sigma_{\mathrm{e}}=\downarrow W_{\mathrm{e}}$. But it will be useful to define $\Vdash$ independently of $W_{\mathrm{m}}, W_{\mathrm{e}}$, assuming only that we have fixed some sets $\Sigma_{\mathrm{m}}$ and $\Sigma_{\mathrm{e}}$ of schedules. The modal base functions $m b_{a}$ (for $a \in\{\mathrm{m}, \mathrm{e}\}$ ) are as in $\S 3$, with $W_{a}$ replaced by $\Sigma_{a}$

$$
\begin{aligned}
m b_{\mathrm{m}}(s, t) & =\left\{s^{\prime} \in \Sigma_{\mathrm{m}} \mid s^{\prime} \approx_{t} s\right\} \\
m b_{\mathrm{e}}(s, t) & =\Sigma_{\mathrm{e}} .
\end{aligned}
$$

However, the inclusion of schedules that are not $\subseteq$-maximal leads to a couple of complications. First, there is the question of persistence. While this is no problem for epistemic modalities, we need to be careful about metaphysical modalities. To hardwire persistence, let us write $\supseteq^{\prime}$ for the restriction of $\supseteq$ to $\Sigma_{\mathrm{m}} \cup \Sigma_{\mathrm{e}}$

$$
s \supseteq s^{\prime} \quad \text { iff } \quad s^{\prime} \subseteq s \text { and } s, s^{\prime} \in \Sigma_{\mathrm{m}} \cup \Sigma_{\mathrm{e}}
$$

and add the quantification $\forall r \supseteq^{\prime} s$ in $^{10}$

$$
s \Vdash t,\langle a\rangle \varphi \quad \text { iff } \quad\left(\forall r \supseteq^{\prime} s\right)\left(\exists s^{\prime} \in m b_{a}(r, t)\right)\left(\exists t^{\prime} \geqslant_{\mathrm{o}} t\right) s^{\prime} \Vdash t^{\prime}, \varphi .
$$

(For $a=\mathrm{e}$ or for world-schedules $s$, the prefix $\left(\forall r \supseteq^{\prime} s\right.$ ) makes no difference; we add it above for the sake of uniformity.) A second complication arises with interpreting the universal modality

$$
s \Vdash t,[a] \varphi \quad \text { iff } \quad\left(\forall r \supseteq \supseteq^{\prime} s\right)\left(\forall s^{\prime} \in m b_{a}(r, t)\right)\left(\exists t^{\prime} \geqslant_{\mathrm{o}} t\right) s^{\prime} \Vdash^{t} t^{\prime}, \varphi
$$

\footnotetext{
${ }^{10}$ This trick (of prefixing $\forall r \supseteq{ }^{\prime} s$ ) is borrowed from a common treatment of negation in $\Vdash$, recalled in the definition of generic sets in the next section.
} 
where the modification $\Vdash^{t}$ on the right hand side takes the partiality of $s^{\prime}$ into account, allowing $s^{\prime}$ to be substituted by an $s^{\prime \prime} \supseteq^{\prime} s^{\prime}$ with $s^{\prime \prime} \approx_{t}$ $s^{\prime}$ to ensure that time is extended sufficiently far beyond $t$

$$
s^{\prime} \Vdash{ }^{t} t^{\prime}, \varphi \quad \text { iff } \quad\left(\exists s^{\prime \prime} \supseteq \varrho^{\prime} s^{\prime}\right)\left(s^{\prime \prime} \approx_{t} s^{\prime} \text { and } s^{\prime \prime} \Vdash t^{\prime}, \varphi\right) .
$$

This complication does not arise in $\langle a\rangle \varphi$, where $s^{\prime}$ is quantified existentially (rather than universally, as in $[a] \varphi$ ) and can absorb the choice of $s^{\prime \prime}$. With the appropriate definitions in place, we can prove Proposition 2 by a routine induction on $\varphi\left(\right.$ for $\Sigma_{\mathrm{m}}=\downarrow W_{\mathrm{m}}$ and $\left.\Sigma_{\mathrm{e}}=\downarrow W_{\mathrm{e}}\right)$.

\section{RECONSTRUCTING WORLDS}

We have arrived at Step 3 of Table 1 . Having defined $\Vdash$ from any two sets $\Sigma_{\mathrm{m}}, \quad \Sigma_{\mathrm{e}}$ of schedules (while suggesting choices of these in Proposition 2 given by $\models$ ), we may ask how to extract $\models$ from $\Vdash$ without assuming $\Vdash$ has been formed from $\models$. Since $\Vdash$ and $\models$ are determined by $\Sigma_{\mathrm{m}}, \Sigma_{\mathrm{e}}$ and $W_{\mathrm{e}}, W_{\mathrm{m}}$ respectively, the question becomes how to define $W_{\mathrm{e}}, W_{\mathrm{m}}$ from $\Sigma_{\mathrm{m}}, \Sigma_{\mathrm{e}}$. Let us record our goal as Proposition 3, leaving open for now just what assumption (Q) and the sets $\uparrow \Sigma_{\mathrm{m}}$ and $\uparrow \Sigma_{\mathrm{e}}$ are.

\section{Proposition 3.}

Given $\Vdash$ with $\Sigma_{\mathrm{m}}, \Sigma_{\mathrm{e}}$ satisfying $(\mathbf{Q})$, let $\models$ be the satisfaction relation given by $W_{\mathrm{m}}=\uparrow \Sigma_{\mathrm{m}}$ and $W_{\mathrm{e}}=\uparrow \Sigma_{\mathrm{e}}$. Then

$$
w, t \models \varphi \quad \text { iff } \quad\left(\exists s \in \Sigma_{\mathrm{m}} \cup \Sigma_{\mathrm{e}}\right) s \subseteq w \text { and } s \Vdash t, \varphi
$$

for all $w \in W_{\mathrm{m}} \cup W_{\mathrm{e}}, t \in \mathrm{T}$, and $\varphi \in \Phi$.

Very roughly, $(\mathbf{Q})$ is the counterpart of the assumption in Proposition 2(b) that $W_{\mathrm{m}} \cup W_{\mathrm{e}}$ is an anti-chain, while $\uparrow$ reverses $\downarrow$. Proposition 3 follows easily if we adopt (14).

(14) Let $\uparrow \Sigma$ be the set of $\subseteq$-maximal elements of $\Sigma$,

$$
\uparrow \Sigma=\left\{s \in \Sigma \mid\left(\forall s^{\prime} \in \Sigma\right) s \subseteq s^{\prime} \text { implies } s^{\prime}=s\right\}
$$

and $(\mathbf{Q})$ stipulate that $\uparrow$ covers $\Sigma_{\mathrm{m}}$ and $\Sigma_{\mathrm{e}}$ in that for all $a \in\{\mathrm{m}, \mathrm{e}\}$ and $s \in \Sigma_{a}$, there exists $s^{\prime} \in \uparrow \Sigma_{a}$ such that $s \subseteq s^{\prime}$.

But $(\mathbf{Q})$ in (14) is a very strong assumption that excludes interesting choices of $\Sigma$. 
Indeed, suppose every schedule in $\Sigma$ were finite, but that each one were properly contained in another one. Then $\uparrow \Sigma$ would be empty, leaving out infinite schedules $\bigcup_{i \geqslant 0} s_{i}$ formed from chains $s_{0} \subset s_{1} \subset s_{2} \subset \ldots$ in $\Sigma$. Such objects $\cup_{i \geqslant 0} s_{i}$ are captured in the usual completeness theorems in logic as maximal consistent extensions. For reasons to be explained shortly, forcing arguments refine the notion of a maximal consistent extension to that of a generic set (e.g. Keisler 1973) - generic not in the sense of 'lions have manes' but rather in connection with $\Vdash$. To spell out this connection, it is useful to extend $\Vdash$ to formulas $\varphi \in \Phi$ with negation $\neg$

$$
s \Vdash t, \neg \varphi \quad \text { iff } \quad\left(\forall r \supseteq^{\prime} s\right) \operatorname{not} r \Vdash t, \varphi
$$

(recalling that $\supseteq$ ' is the restriction of $\supseteq$ to $\Sigma_{\mathrm{m}} \cup \Sigma_{\mathrm{e}}$ ). Now, $G$ is generic if

(i) for all $s \in G$ and $s^{\prime} \in \Sigma_{\mathrm{m}} \cup \Sigma_{\mathrm{e}}, s^{\prime} \subseteq s$ implies $s^{\prime} \in G$

(ii) every pair $s, s^{\prime} \in G$ has a common extension $s^{\prime \prime} \in G$ : $s^{\prime \prime} \supseteq s \cup s^{\prime}$

(iii) for all $\varphi \in \Phi$ and $t \in \mathrm{T}$, there is an $s \in G$ such that either $s \Vdash t, \varphi$ or $s \Vdash t, \neg \varphi$.

Conditions (i) and (ii) essentially make $G$ an ideal. What differentiates generic sets from plain maximal consistent extensions is condition (iii), as illustrated by the following example. Over the time intervals

$$
\mathrm{TI}=\{(0,1),(1,2),(2,3), \ldots\}
$$

and eventuality descriptions

$$
\mathrm{ED}=\{\operatorname{die}(\text { Socrates }), \text { alive }(\text { Socrates })\},
$$

let us define the schedules $s_{0}=\emptyset$ and for $i \geqslant 0$,

$$
\begin{aligned}
s_{i+1} & =s_{i} \cup\{((i, i+1), \text { alive }(\text { Socrates }))\} \\
s_{i}^{\prime} & =s_{i} \cup\{((i, i+1), \operatorname{die}(\text { Socrates }))\} .
\end{aligned}
$$

Suppose these schedules constituted $\Sigma_{\mathrm{m}}$ and $\Sigma_{\mathrm{e}}$

$$
\begin{aligned}
\Sigma_{\mathrm{m}} & =\left\{s_{i} \mid i \geqslant 0\right\} \cup\left\{s_{i}^{\prime} \mid i \geqslant 0\right\} \\
& =\Sigma_{\mathrm{e}} .
\end{aligned}
$$

Clearly, Socrates is immortal in the maximal consistent extension $\bigcup_{i \geqslant 0} s_{i}$

$$
(\forall t \in \operatorname{TI})(\exists i \geqslant 0) s_{i}(t, \text { alive(Socrates) }) .
$$


On the other hand, applying condition (iii) to $\varphi=[\mathrm{m}] \operatorname{die}($ Socrates) and $t=(0,1)$, it follows from

$$
(\ddagger) \quad\left(\forall s \in \Sigma_{\mathrm{m}}\right)\left(\exists t^{\prime} \in \mathrm{TI}\right) s \cup\left\{\left(t^{\prime}, \operatorname{die}(\text { Socrates })\right)\right\} \in \Sigma_{\mathrm{m}}
$$

that Socrates must die in every generic set $G$

$$
\left(\exists t^{\prime} \in \operatorname{Tr}\right)(\exists s \in G) s\left(t^{\prime}, \operatorname{die}(\text { Socrates })\right) .
$$

This is not to say that generic sets commit us to the mortality of Socrates. Only that if we want to entertain the metaphysical possibility that Socrates is immortal in a generic set, then we had better choose $\Sigma_{m}$ so that ( $\ddagger$ ) fails. (This is easy enough to arrange say, by including infinite schedules in $\Sigma_{\mathrm{m}}$, or by introducing an eventuality description immortal(Socrates) with a suitable constraint on its schedules in $\Sigma_{\mathrm{m}}$.)

Armed with the notion of a generic set, we can devise more useful choices of $\uparrow$ and (Q) for Proposition 3 than (14). For all $\Sigma \subseteq \Sigma_{\mathrm{m}} \cup \Sigma_{\mathrm{e}}$, let $\uparrow \Sigma$ form the unions of generic sets contained in $\Sigma$

$$
\uparrow \Sigma=\{\cup G \mid G \text { is generic, and } G \subseteq \Sigma\},
$$

the intuition being that for $a \in\{\mathrm{m}, \mathrm{e}\}$, a generic set $G$ contained in $\Sigma_{a}$ induces the $a$-world $\cup G$. As for $(\mathbf{Q})$, we take it to say

$$
\begin{aligned}
\uparrow\left(\Sigma_{\mathrm{m}} \cup \Sigma_{\mathrm{e}}\right)= & \left(\uparrow \Sigma_{\mathrm{m}}\right) \cup\left(\uparrow \Sigma_{\mathrm{e}}\right) \text { and } \\
& \text { ED and TI are finite or countable. }
\end{aligned}
$$

Under these assumptions, Proposition 3 can be proved along standard lines in forcing, using the persistence of $\Vdash$ (Proposition 2(a) above) and the fact that every $s \in \Sigma_{\mathrm{m}} \cup \Sigma_{\mathrm{e}}$ belongs to a generic set, provided $\mathrm{TI} \times \mathrm{ED}$ is finite or countable (see e.g. Lemma 1.4 in Keisler 1973, page 101). Two other facts, (15) and (16), are worth recording.

(15) The forcing of doubly negated formulas

$$
s \Vdash t, \neg \neg \varphi \quad \text { iff } \quad\left(\forall r \supseteq^{\prime} s\right)\left(\exists p \supseteq^{\prime} r\right) p \Vdash t, \varphi
$$

reduces (as usual) to generic sets

$$
s \Vdash t, \neg \neg \varphi \quad \text { iff } \quad(\forall \text { generic } G \text { such that } s \in G) \cup G \vDash t, \varphi .
$$

(16) If $W_{\mathrm{m}} \cup W_{\mathrm{e}}$ is an anti-chain, then

$$
\uparrow \downarrow W=W \quad \text { for } \quad W \in\left\{W_{\mathrm{m}}, W_{\mathrm{e}}, W_{\mathrm{m}} \cup W_{\mathrm{e}}\right\}
$$


where $\uparrow$ is defined relative to $\Sigma_{\mathrm{m}}=\downarrow W_{\mathrm{m}}$ and $\Sigma_{\mathrm{e}}=\downarrow W_{\mathrm{e}}$.

Concerning (15), note that

$$
s \Vdash t, \varphi \quad \text { implies } \quad s \Vdash t, \neg \neg \varphi
$$

but that in general, the converse fails (and hence so would the last equivalence in (15), if we were to drop $\neg \neg$ from its left hand side). As for (16), what it says essentially is that if we feed our machinery a set of worlds (that is, an anti-chain $W_{\mathrm{m}} \cup W_{\mathrm{e}}$ ), then we get it back. No more, no less. But of course, what makes $\Vdash$ and genericity interesting is that worlds are not, at the outset, required. Notice that in the Socrates example of this section, no schedule in $\Sigma_{\mathrm{m}}=\Sigma_{\mathrm{e}}$ is a world (i.e. $\subseteq$-maximal).

\section{CONCLUSION}

We have carried out Steps 1-3 of Table 1, reformulating the temporal properties of CoN2 in terms of a forcing relation

$$
s \Vdash t, \varphi
$$

involving schedules $s$ that may or may not be worlds. But why bother with a schedule $s^{\prime}$ forcing $t, \varphi$ that is $\subset$-smaller than a world $w$ ? Because $s^{\prime}$ picks out more schedules that force $t, \varphi$ than $w$. By the persistence of $\Vdash$, any schedule bigger than $s^{\prime}$ must force $t, \varphi$, including $w$, all schedules bigger than $w$ (of which there are none, if $w$ is a world), and other worlds bigger than $s^{\prime}$ (of which there may be any number). In other words, small is beautiful. We should try to make a schedule $s$ that forces $t, \varphi$ smaller (and not only bigger, as we do when forming worlds via generic sets).

With this in mind, let us close by considering the prospects of truncating schedules. More precisely, given a schedule $s$ and time $t$, let $s_{t}$ be the restriction of $s$ to times $<$ or $\subseteq t$

$$
s_{t}=\left\{\left(t^{\prime}, P\right) \in s \mid t^{\prime}<t \text { or } t^{\prime} \subseteq t\right\}
$$

The question is: for which $\varphi \in \Phi$ can we count on (17)?

(17) $s \Vdash t, \varphi \quad$ iff $\quad s_{t} \Vdash t, \varphi$

(17) goes through without a hitch for nearly all the clauses of $\Vdash$. The only problematic cases are the metaphysical modalities $\langle\mathrm{m}\rangle \varphi$ and $[\mathrm{m}] \varphi$, 
which can be saved if we strengthen the prefix $\forall r \supseteq^{\prime} s$ to $\forall r \supseteq^{\prime} s_{t}$, yielding

$$
s \Vdash t,\langle\mathrm{~m}\rangle \varphi \quad \text { iff } \quad\left(\forall r \supseteq^{\prime} s_{t}\right)\left(\exists s^{\prime} \in m b_{\mathrm{m}}(r, t)\right)\left(\exists t^{\prime} \geqslant_{\mathrm{o}} t\right) s^{\prime} \Vdash t^{\prime}, \varphi
$$

and

$$
s \Vdash t,[\mathrm{~m}] \varphi \quad \text { iff } \quad\left(\forall r \supseteq^{\prime} s_{t}\right)\left(\forall s^{\prime} \in m b_{\mathrm{m}}(r, t)\right)\left(\exists t^{\prime} \geqslant_{\mathrm{o}} t\right) s^{\prime} \Vdash{ }^{t} t^{\prime}, \varphi \text {. }
$$

With this modification, ${ }^{11}$ we secure (17) for all $\varphi \in \Phi$, without (remarkably) losing any of our previous results (including Propositions 2 and 3, and (15),(16)). Moreover, suppose we introduce a fresh symbol $R \notin \mathrm{ED}$ (for Reichenbach's reference time) and define an $R$-sked to be a relation of the form

$$
s[t]=s_{t} \cup\{(t, R)\}
$$

for some schedule $s$ and time $t$. Then we can encode $\Vdash$ in statements s $\triangleright \varphi$ (pronounced "s pins $\varphi$ ") between $R$-skeds s and formulas $\varphi$ satisfying (18). (18) $s[t] \triangleright \varphi \quad$ iff $\quad s \Vdash t, \varphi$

To construe (18) as a definition of $\triangleright$, we need to show that the choice there of $s$ and $t$ for the sked $\mathrm{s}=s[t]$ is immaterial - that is, whenever $s[t]=s^{\prime}\left[t^{\prime}\right]$,

$$
s \Vdash t, \varphi \quad \text { iff } \quad s^{\prime} \Vdash t^{\prime}, \varphi \text {. }
$$

But that is immediate from (17) and $R \notin$ ED. Alternatively (instead of deriving $\triangleright$ from $\Vdash$ ), we might define $\triangleright$ from scratch $^{12}$ and read (18) from right to left as a definition of $\Vdash$ from $\triangleright$. This route to $\Vdash$,

\footnotetext{
${ }^{11}$ As before, the prefix $\forall r \supseteq^{\prime} s_{t}$ has no effect on the epistemic modalities, so we can replace $\mathrm{m}$ above by $a \in\{\mathrm{m}, \mathrm{e}\}$ for the sake of uniformity.

12 This is easy, albeit tedious. Given an $R$-sked s, let us write last(s) for the unique time $t$ such that $\mathrm{s}(t, R)$, and write $\mathrm{s}_{R}$ for the schedule $\mathrm{s}-\{(\operatorname{last}(\mathrm{s}), R)\}$ obtained from $\mathrm{s}$ by removing $R$. Then

$$
\begin{array}{rll}
\mathrm{s} \triangleright P & \text { iff } & \mathrm{s}(\operatorname{last}(\mathrm{s}), P) \quad \text { for } P \in \mathrm{ED} \\
\mathrm{s} \triangleright \varphi \subseteq & \text { iff } & (\exists t \in \operatorname{domain}(\mathrm{s})) t \subseteq \operatorname{last}(\mathrm{s}) \text { and } \mathrm{s}_{R}[t] \triangleright \varphi \\
\mathrm{s} \triangleright \operatorname{Perf}(\varphi) & \text { iff } & (\exists t \in \operatorname{domain}(\mathrm{s})) t<\operatorname{last}(\mathrm{s}) \text { and } \mathrm{s}_{R}[t] \triangleright \varphi \\
\mathrm{s} \triangleright\langle a\rangle \varphi & \text { iff } & \left(\forall \mathrm{r} \supseteq^{\prime} \mathrm{s}\right)\left(\exists \mathrm{s}^{\prime} \in m b_{a}^{\mathrm{o}}(\mathrm{r}, \text { last }(\mathrm{s}))\right) \mathrm{s}^{\prime} \triangleright \varphi \\
\mathrm{s} \triangleright[a] \varphi & \text { iff } & \left(\forall \mathrm{r} \supseteq^{\prime} \mathrm{s}\right)\left(\forall \mathrm{s}^{\prime} \in m b_{a}^{\mathrm{o}}(\mathrm{r}, \text { last }(\mathrm{s}))\right)\left(\exists \mathrm{s}^{\prime \prime} \approx_{\text {last }(\mathrm{s})} \mathrm{s}^{\prime}\right) \\
& & \text { last }\left(\mathrm{s}^{\prime \prime}\right) \geqslant \text { last }(\mathrm{s}) \text { and } \mathrm{s}^{\prime \prime} \triangleright \varphi
\end{array}
$$
}

where

$$
m b_{a}^{\mathrm{o}}(\mathrm{s}, t)=\left\{s^{\prime}\left[t^{\prime}\right] \mid s^{\prime} \in m b_{a}\left(\mathrm{~s}_{R}, t\right) \text { and } t^{\prime} \geqslant_{\mathrm{o}} t\right\} .
$$


however, depends on (17), which may well fail for $\varphi$ incorporating the progressive. ${ }^{13}$

\section{Acknowledgments}

I am indebted to Cleo Condoravdi, Stefan Kaufmann and Frank Veltman for helpful comments, and to two anonymous referees for thoughtful criticisms. My thanks also to the organizers and participants of the 7th Symposium on Logic and Language in Pecs, Hungary (August 2002), where an early version of this paper (here superseded) was presented under the shameless title 'Between events and worlds under historical necessity.'

TIM FERNANDO

Computer Science Department

Trinity College, Dublin 2

Ireland

e-mail:Tim.Fernando@cs.tcd.ie
Received: 02.03 .03

Final version received: 28.08 .04

\section{REFERENCES}

Abusch, D. (1998) 'Generalizing tense semantics for future contexts'. In S. Rothstein, (ed.), Events and Grammar, Kluwer. Dordrecht; 13-33.

Condoravdi, C. (2002) 'Temporal interpretation of modals: Modals for the present and for the past'. In D. Beaver, S. Kaufmann, B. Clark, \& L. Casillas, (eds), The Construction of Meaning. CSLI. Stanford, 59-88.

Dowty, D. R. (1979) Word Meaning and Montague Grammar. Reidel. Dordrecht.

Fernando, T. (2003) 'Reichenbach's E, R and $\mathrm{S}$ in a finite-state setting. Sinn und Bedeutung 8 (Frankfurt), (www.cs.tcd. ie/Tim.Fernando/wien.pdf)

van Fraassen, B. C. (1969) 'Facts and tautological entailments'. Journal of Philosophy, 66(15):477-487.
Gennari, S. P. (2003). 'Tense meanings and temporal interpretation'. Journal of Semantics, 20(1):37-71.

Keisler, H. J. (1973) 'Forcing and the omitting types theorem'. In M. Morley, (ed.), Studies in Model Theory. The Mathematical Association of America, 96-133.

Plotkin, G. (1983) Domains ('Pisa notes'). Department of Computer Science, University of Edinburgh.

Schubert, L. (2000) 'The situations we talk about'. In J. Minker, (ed.), LogicBased Artificial Intelligence. Kluwer. Dordrecht, 407-439.

Steedman, M. The Productions of Time. Draft, ftp://ftp.cogsci.ed.ac.uk/pub/ steedman/temporality/temporality.ps. gz, July 2000. (Subsumes 'Temporality', in

${ }^{13}$ Details in Fernando (2003), where schedules are reduced further to regular languages over the alphabet $\operatorname{Pow}(\mathrm{ED} \cup\{R, S\})$, with ED assumed finite, towards a finite-state formulation of ideas described in Steedman 2000. 
J. van Benthem \& A. ter Meulen, (eds), Veltman, F. (1984) 'Data semantics'. In Handbook of Logic and Language. Elsevier J. Groenendijk, T.M.V. Janssen \& M. North Holland, 895-935,1997.)

Thomason, R. (1984) 'Combinations of tense and modality'. In D. Gabbay \& F. Guenthner, (eds), Handbook of Philosophical Logic. Reidel, 135-165. Stokhof, (eds), Truth, Interpretation and Information, Foris, 43-63.

Veltman, F. (1996) 'Defaults in update semantics'. Journal of Philosophical Logic 25:221-261. 